\title{
Microcontroller based Intelligent Digital Volume Controller with Timer
}

\author{
Diptarup Paul \\ Department of Computer Science and Engineering \\ JIS College of Engineering \\ Kalyani, Nadia-741235, West Bengal, India
}

\author{
Sukalyan Som \\ Department of Computer Science \\ Barrackpore Rastraguru Surendranath College \\ 85, Middle Road \& 6, Riverside Road, Kolkata- 120
}

\begin{abstract}
In this paper a Microcontroller based intelligent digital volume controller is presented to control music system and PC speaker volume digitally and change volume levels automatically in case of certain predefined incidents such as incoming or outgoing telephone call and pre-set alarm. The device is constructed with AT89S52 microcontroller, from ATMEL family with 8051 architecture, and its software program being written in $\mathrm{C}$ language.
\end{abstract}

\section{Keywords}

Microcontroller, AT89S52, CD4066, optocoupler, Zener diode, Seven-segment display, voltage divider.

\section{INTRODUCTION}

The project is aimed at being a regular household product. This not only has a digitalized volume control mechanism of music system but also includes intelligent decision making to change the volume level for certain incidents. For how many times we have faced inconvenience to attend a phone call when music is playing loud in our room? Well, this device brings the solution. It automatically tracks the telephone line and detects an incoming call, and brings down the volume to a much lower definable level. Same thing happens for outgoing calls too. And yes, this can be done manually as well!

The device also contains a clock, implemented with computer software, by which we can set an alarm, and also set different volume levels for a particular time of the day. That means an alarm clock, but with a brain

For example: Let the time be set at $10 \mathrm{PM}$ in the clock, the clock is there to decrease the volume at $10 \mathrm{PM}$, without ones interference, keeping one busy on what one is up to.

In case a user wants to use this device with his computer speaker system there is an option for him to manage the alarm system from the computer itself. Yes! This device can be controlled from the computer by the means of serial communication.

\section{BASIC TERMINOLOGY}

A micro controller is a small computer on a single integrated circuit containing a processor core, memory, and programmable input/output peripherals where peripherals can be any electronic device.

As the name suggests, the device is controlled by a microcontroller, AT89S52 [1], from Atmel Corporation.

AT89S52[1] (see Figure 1), is a 40 pin low-power, highperformance 8-bit microcontroller with 8 kilo bytes of insystem programmable Flash memory, 256 bytes of Random Access Memory, 32 I/O lines, Watchdog timer, two data pointers, three 16-bit timer/counters, a six-vector two-level interrupt architecture, a full duplex serial port, on-chip oscillator, and clock circuitry.

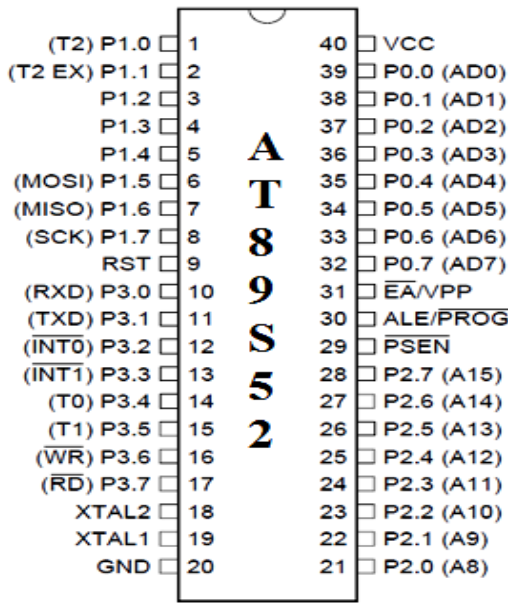

Fig 1: Pin Configuration of AT89S52.

Volume levels are being switched by the help of analogue switching IC, CD4066. CD6044 is a quad bilateral switch[2], i.e. it consists of four independent bilateral switches. A single control signal is required per switch. Both the $\mathrm{p}$ and $\mathrm{n}$ device in a given switch are biased ON or OFF simultaneously by the control signal. As shown in Figure 2, the well of the n-channel device on each switch is either tied to the input when the switch is ON or to VSS when the switch is OFF.

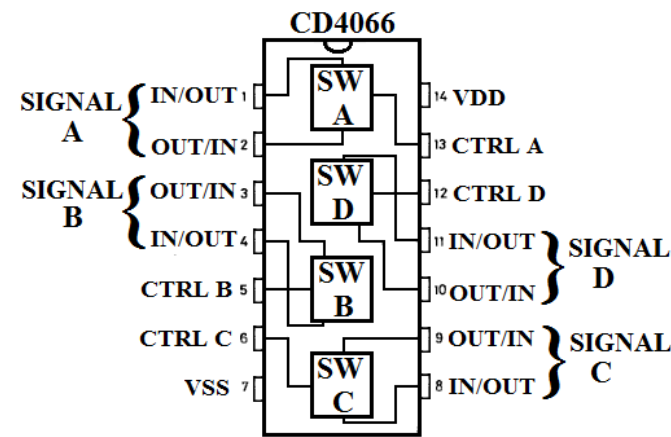

Fig 2: Schematic diagram of CD4066.

The device is being switched though the telephone line by the help of an optocoupler (also called opto-isolator, phototransistor). It is an electronic device designed to transfer electrical signals by utilizing light waves to provide coupling with electrical isolation between its input and output. The main purpose of an opto-isolator is to prevent high voltages or 
rapidly changing voltages on one side of the circuit from damaging components or distorting transmissions on the other side [3]. The working is shown in Figure 3, where side 1, 2 acts as input, i.e. anode and cathode respectively and 3, 4 as output, i.e. collector and emitter respectively.

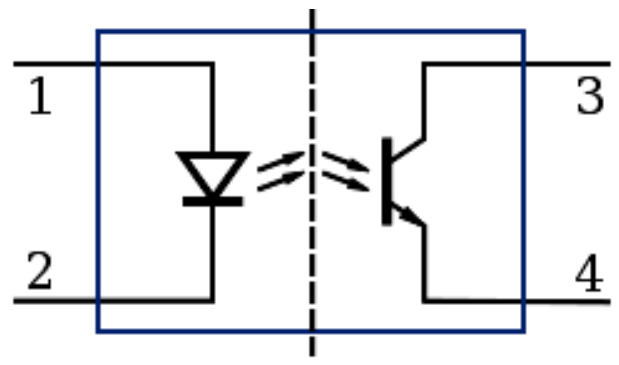

Fig 3: Schematic of an optocoupler.

The standard voltage for the telephone system is 48 volts DC. Ringing voltage is much higher, around 90 volts $\mathrm{AC}$ at a low frequency. So the opto-isolator prevents the main circuit from this high voltage and also helps in switching when the phone rings.

A Zener diode, or breakdown diode is a special kind of diode which allows current to flow in the forward direction in the same manner as an ideal diode, but will also permit it to flow in the reverse direction when the voltage is above a certain value known as the breakdown voltage, "Zener knee voltage" or "Zener voltage"[4]. Break down diodes Are p-ndiodes operated in the breakdown region of the reverse voltage characteristics [5]. In this circuit a 13 volte zener diode has been used in the telephone line before the optocoupler to ensure that it only acts for voltage levels more than 13 volts, i.e. the condition when the telephone receiver is down.
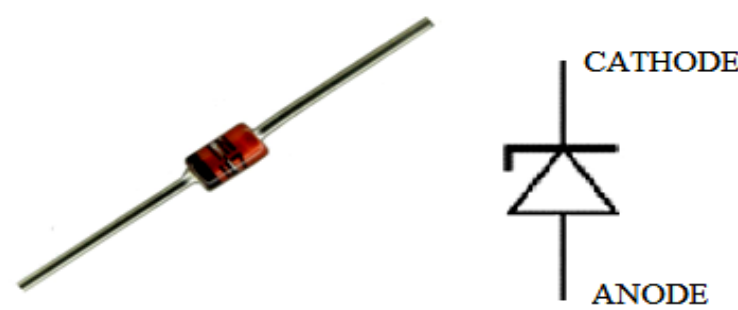

Fig 4: Zener diode.

In the above figure (Figure 4), a typical Zener diode has been represented.

The volume level and other options are being displayed in a 7 segment display. Seven-segment displays are widely used in digital clocks, electronic meters, and other electronic devices for displaying numerical information. It contains 7 LEDs arranged in such a manner that when illuminated accordingly, can display numerical digits. It is of two types, common anode and common cathode. The schematic of a 7 segment display is shown in Figure 5. Common anode 7 segment display is used in this project.
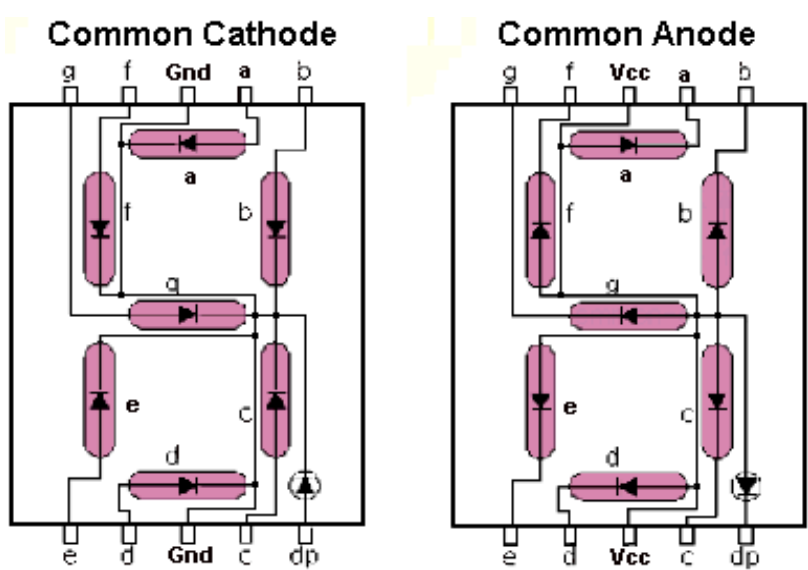

Fig 5: Schematic view of a 7 segment display.

The volume levels are defined by the help of series voltage dividers. In electronics, a voltage divider (also known as a potential divider) is a simple linear circuit that produces an output voltage $\left(\mathrm{V}_{\text {out }}\right)$ that is a fraction of its input voltage $\left(\mathrm{V}_{\mathrm{in}}\right)$. Voltage division refers to the partitioning of a voltage among the components of the divider [6]. Figure 6 is a representation of a voltage divider.

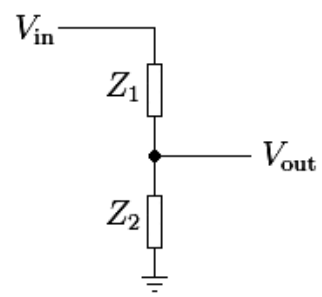

Fig 6: Voltage divider

\section{PROPOSED LAYOUT}

\subsection{Idea about the project}

The purpose of this project is to develop a digital volume controlling device that is capable of controlling the volume of a music system and PC speaker system to a predefined volume level when the user is receiving a phone call in his land phone. There will be provision for the user to control the device from the computer if he wants to. Moreover the user will be given a option to adjust the volume to a predefined level at a particular time of the day by the clock implemented in the computer software provided along with the device.

\subsection{Presently available options}

Presently, available volume controllers with timer are mainly software based, i.e. they are usable in a computer only and no hardware implementable volume controller with telephone based intelligence is available. For example, Alarm[7], Cool Timer[8], Timeleft [9] etc.

The existing softwares mentioned here have the following generic features.

a. They act as a reminder on an oncoming event.

b. Tracks the amount of time left for specified event.

c. They are capable of being a countdown timer and sound an alarm when the alarm goes off.

Existing softwares provide alarm facility by means of software and are meant explicitly for PC use. The volume levels cannot be controlled automatically by the softwares users' choice 
Thus it can be cited that, this device has the following edge over the existing systems and/or software.

a. This is a general purpose digital volume controller for both music system and PC.

b. It is an attempt to combine volume control with alarm by the means of hardware.

c. It is a novel attempt that includes automatic volume level changes in case of special incidents such as incoming telephone call and also outgoing call attempts.

d. Volume levels can be set by the user, according to the need.

\subsection{Working principle}

The main system runs on a AT89S52[1] microcontroller, with a 7 segment display to display the volume level, with 4 analogue switches, CD41066, two for each channel, left channel and the right giving us 8 defined sound levels. The telephone line signaling if fed to the microcontroller through a optocoupler. The serial communication procedure is implemented for the communication with the computer by the means of a USB to serial converter [10]. Switches for increasing and decreasing volume digitally, muting audio, telephone interfacing, alarm interfacing, volume defining are being supplied. The following Figure 7 is a block diagrammatical representation of the project plan.

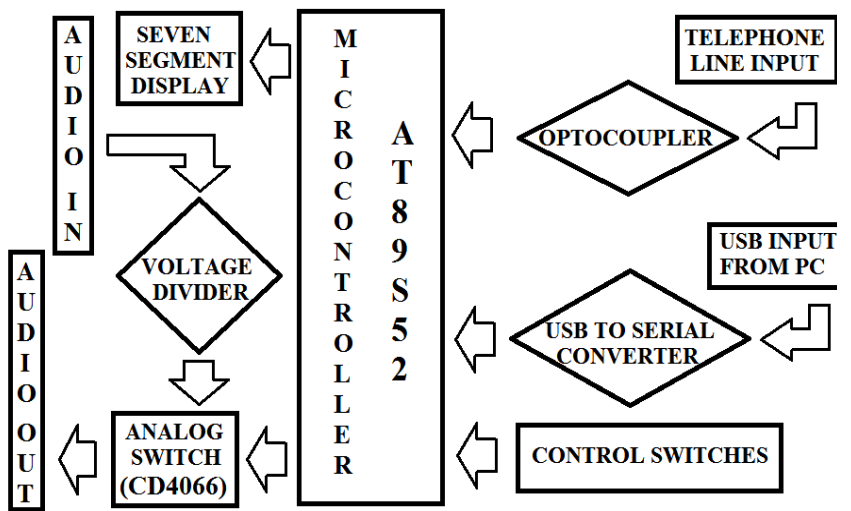

Fig 7: Volume control system functional block diagram.

The sound level switching is implements by the means of voltage dividing with resistors attached in series and having input audio signal at one end and GND at the other.

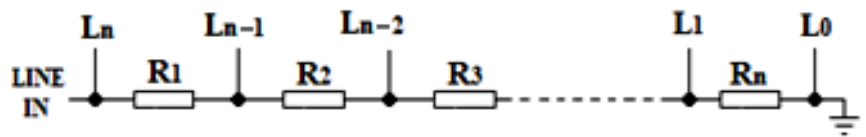

Fig 8: Sound level switching implementation block diagram.
The block diagrammatical representation of sound level switching is shown in the above figure (see Figure 8). Where $\mathrm{L}_{0}$ is the mute condition and $\mathrm{L}_{\mathrm{n}}$ is the highest volume level. $R_{1}, R_{2}, R_{3}, \ldots, R_{n}$ are resistors of equal value. In this project eight resistors are being used against each of channel left and right. To get $8+1$ i.e. 9 distinct sound levels.

\subsection{Circuital implementation and control description}

The working model of the device is made a quite user friendly. The circuit diagram is shown in Figure 9. Switches S1 to S6 are configured as VOLUME UP, VOLUME DOWN, LOW, LOW SET, TEL VOL and TEL BREAK respectively.

VOLUME UP key is programmed to increase the volume of the system, as because this system comes with a built-in volume controller.

VOLUME DOWN key is programmed to decrease the volume of the system.

TEL VOL key is programmed to set the volume of the speaker system, i.e. the volume level that the speaker system will be playing in, when the telephone is in use. It can be ' 0 ' i.e. mute, or any level user wants it to be.

LOW SET is to set the volume level of the LOW button operation, if in this case the volume level is set to 0 , it can act as mute button, depending upon the user's will.

TEL BREAK button is to bypass the telephone interfacing. If when the music is silenced by the device and the user wants to resume it to the volume it was playing, pressing this button will bypass the silenced condition till the end of the call.

A telephone interfacing female socket, RJ11 is attached to the telephone input part of the circuit.

Audio sockets are attached at LINE IN and LINE OUT. S7 is a DPDT switch to bypass the audio line from the circuit. A USB to Serial converter [10] connected to this device for serial communication with a computer.

The functional description, in other words, the user manual to operate the system, is stated as follows,

a. A telephone interfacing female socket, RJ11 is attached to the telephone input part of the circuit.

b. Audio sockets are attached at LINE IN and LINE OUT. S7 is a DPDT switch to bypass the audio line from the circuit. A USB to Serial converter [10] connected to this device for serial communication with a computer. 


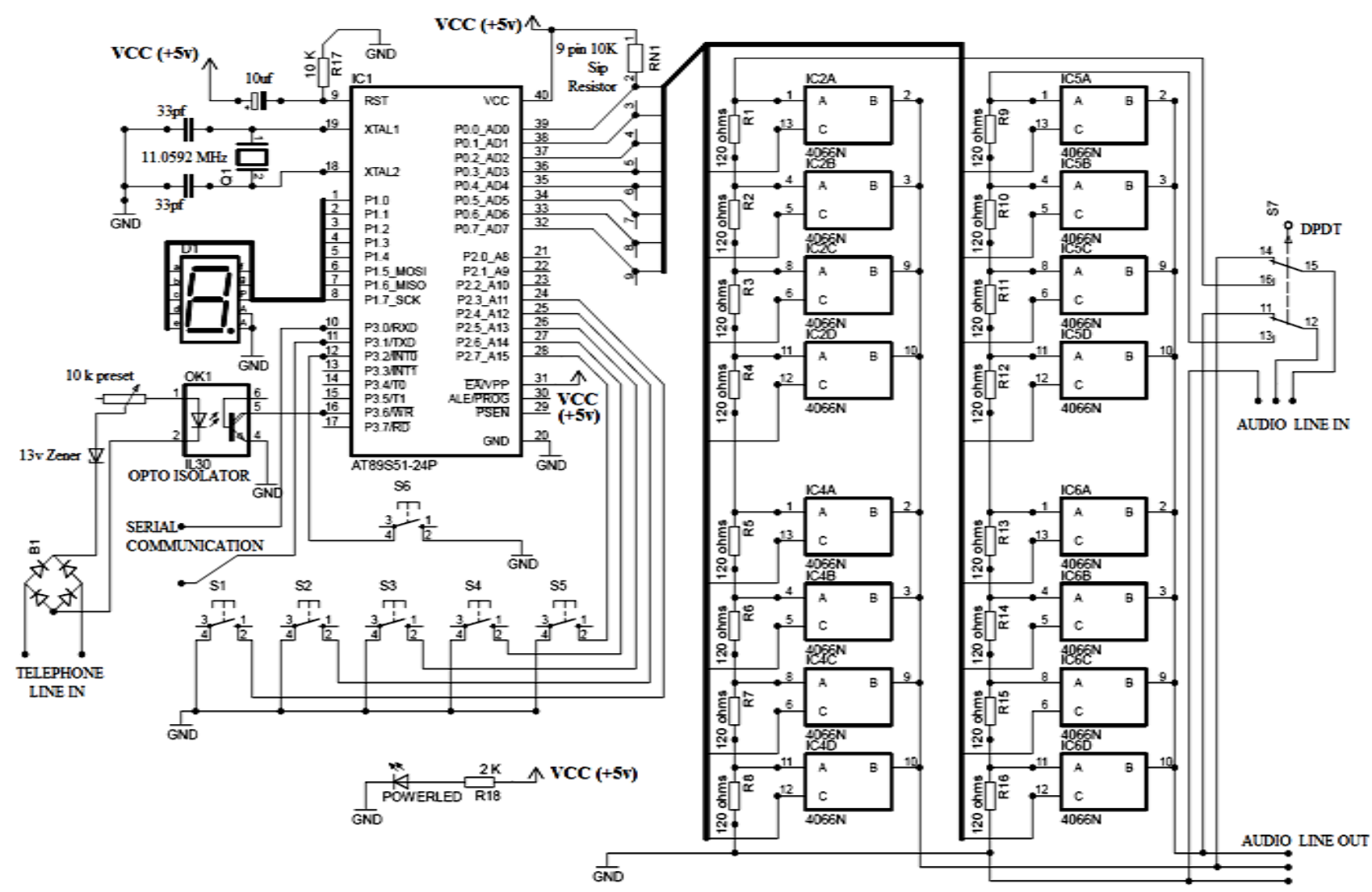

Fig 9: Circuit Diagram

c. The device is connected to PC or any audio device and the speakers are connected to this device.

d. Telephone line is connected to the RJ 11 socket in parallel to the main telephone line.

e. Audio input is taken and set in to the voltage divider, created by resistances in series.

f. From every point in the voltage divider ladder, output is taken.

g. The outputs are channeled to the Analogy Switching IC CD4066 as input.

h. The switches in CD4066 are driven by the microcontroller AT89S52[1].

i. Volume can be increased or decreased up pressing VOLUME UP and VOLUME DOWN button respectively.

j. The present volume level is displayed in the 7-Segment Display.

k. On pressing the LOW button, the volume is decreased to a certain pre-defined level, and on pressing it for the second time or UP or DOWN buttons, swaps back to the earlier set volume level.

1. The volume level for this case can be defined by pressing the LOW SET button, which is pre-defined to 1 . The volume level shown in the 7-segment display is now set to be used as the low. To set this level, the user has to take the volume level to the level he needs to set as LOW, by pressing the VOLUME UP and VOLUME DOWN keys and press the LOW SET key. m. If the present volume level is lower than the 'low set value', the LOW button is not going to work.

n. To denote the LOW state, the 7-Segment Display alternately glows ' $\mathrm{Dp}$ ' and 'the set level'.

o. When the telephone receiver is picked up, for receiving a call or to make a call, the volume level jumps to a predefined level 1 , or a user defined value.

p. The volume level moves back to normal when the telephone receiver is put back into place. After completing the call.

q. To set the volume level during the telephone call, the TELVOL button is pressed after taking the volume level to the desired volume level being shown in the 7 segment display, while the telephone line is not being used. This volume level is now set to be used during the call.

r. To denote the land phone call state, the 7-Segment Display alternately glows 'g' LED and 'the set level'.

s. In case of parallel connection in ones' home, the telephone interference can be bypassed by pressing the TEL BREAK key, which resets itself with the end of the call.

t. The telephone interference can be recalled by pressing the TEL BREAK button again while the telephone line is being used.

u. This device can be operated from the PC itself and in that case, the PC can act as the timer. In this case one can set 
the time to either increase or decrease the volume to the level, set by the LOW SET button.

The device runs on 5 volts DC. To power this device one can use a DC adaptor ranging from 6 to 15 volts. The voltage is stepped down to 5 volts be the use of a voltage regulating IC LM317.]

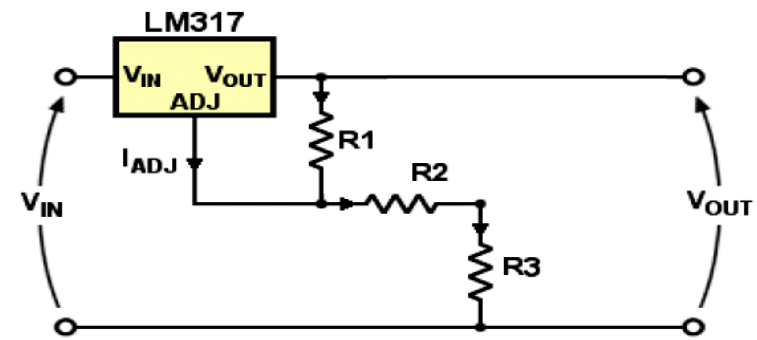

Fig 10: LM317 voltage regulation block diagram. In the above diagram (Figure 10) voltage regulating implementation with IC LM317 is shown which is used to generate 5 voltage for the circuit. Here R1 is $220 \mathrm{ohms}$, R2 is
100 ohms and R3 is 560 ohms. $\mathrm{V}_{\text {IN }}$ can be any voltage between $6 \mathrm{~V}$ to $15 \mathrm{~V}$. VOUT is $5 \mathrm{~V}$. The datasheet of LM317 [11] and online calculator[12] has been consulted for the values of $\mathrm{R} 1, \mathrm{R} 2$ and $\mathrm{R} 3$.

\subsection{Flow chart}

The flow chart follows in Figure 11.

\section{SOFTWARE INTERFACE}

This device comes with a computer software. This interface has been developed with Microsoft Visual Basic 6.0. This software is optional to a user. The privilege a user gets, while using this software is that, he gets the option to set timer to control the device. This timer helps him to switch the device to LOW volume level, i.e. the operation he gets by pressing the LOW key in the hardware. But in this case the software is there to make him lazy. The only thing he has to do is to plug the device to his computer USB port, run this software and set the time. The software will do the rest. The following figure (see Figure 12) shows a screen shot of the software in action.

\section{Volume timer}

Current time: $23: 15: 27$

Present Volume Level: 7

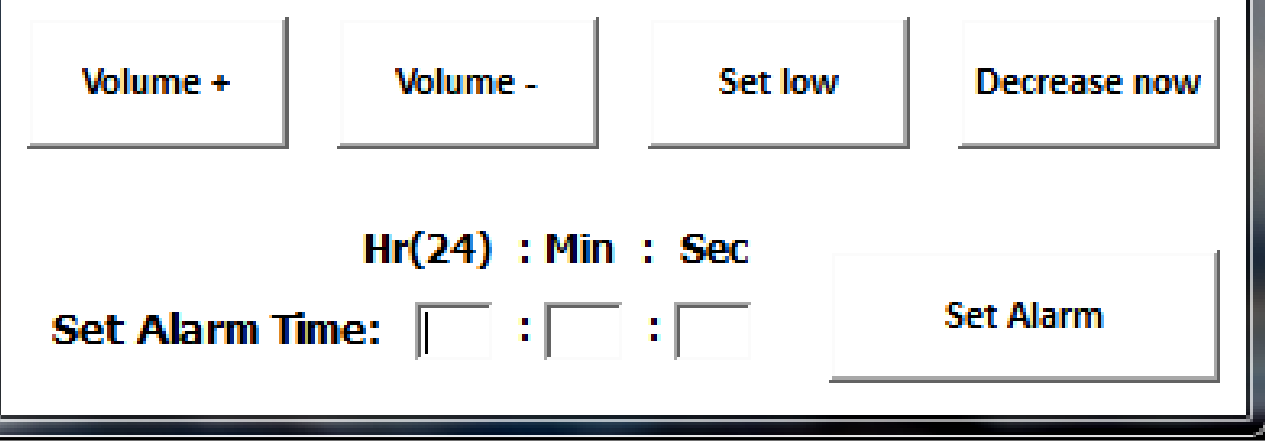

Fig 12: Software interface.

The functionality of each button of the software is stated as follows,

Volume+ - This button will increase the volume level in the device by level 1, the same way the VOLUME UP key acts in the hardware device.

Volume- - This button will decrease the volume level in the device be level 1, the same way the VOLUME DOWN key acts in the hardware device.

Set low - Thus button will set the volume level of the low volume level same as LOW SET in the hardware.

Decrease now - This button will decrease the volume level to LOW. The functionality of the button is same as that of the LOW key in the hardware.

Current time - It shows the system time in 24 hour format from the computer.

Present Volume Level - This shows the volume level the hardware is set to. It is volume level which is being displayed in the 7-segment display in the hardware.
Set Alarm Time - This is the text area where the user is required to type in the time in hour, minute, and second in HH:MM:SS format.

Set Alarm - This button sets the time entered in the text areas of Set Alarm Time. If any of the text boxes are left as blank by the user then it takes default value as 0 . At this particular time the device will switch itself to LOW state and vice versa, provided the device has to remain connected to computer. 


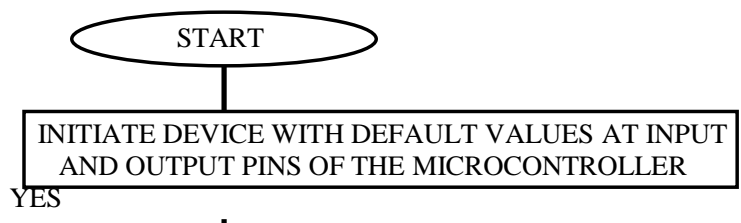

\section{INTIATE SERIAL COMMUNICATION}

KEPT

NITIATE A VOLUME LEVEL COUNTER AT A DEFAULT VALUE

$$
\begin{array}{ll|l|}
\multicolumn{1}{c|}{\text { LOOP BEGIN }} \\
\text { NO }
\end{array}
$$

CHECK KEYPAD FOR INPUT AND SERIAL PORT FOR DATA
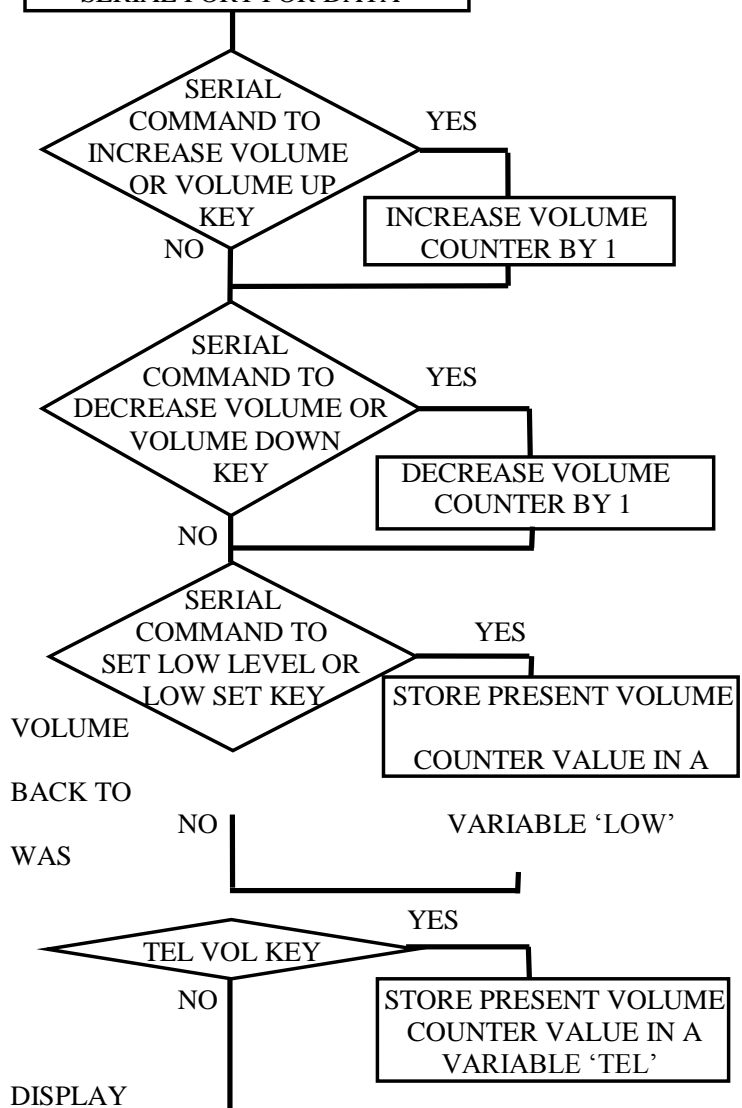

DISPLAY

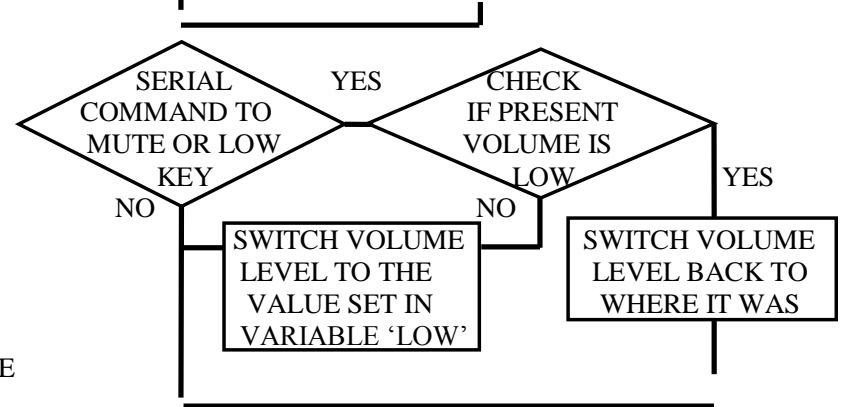

COMMUNICATION.

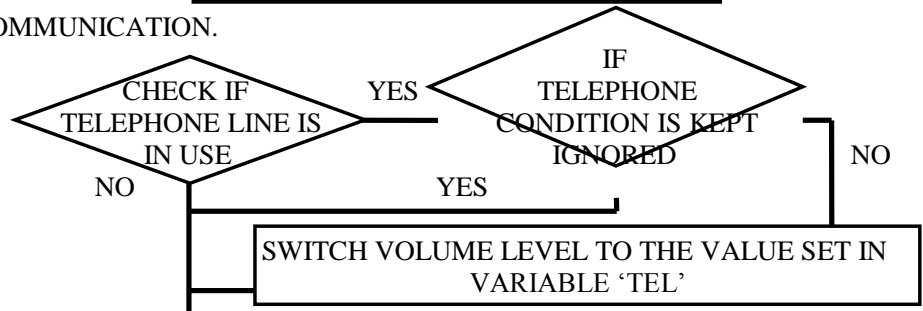

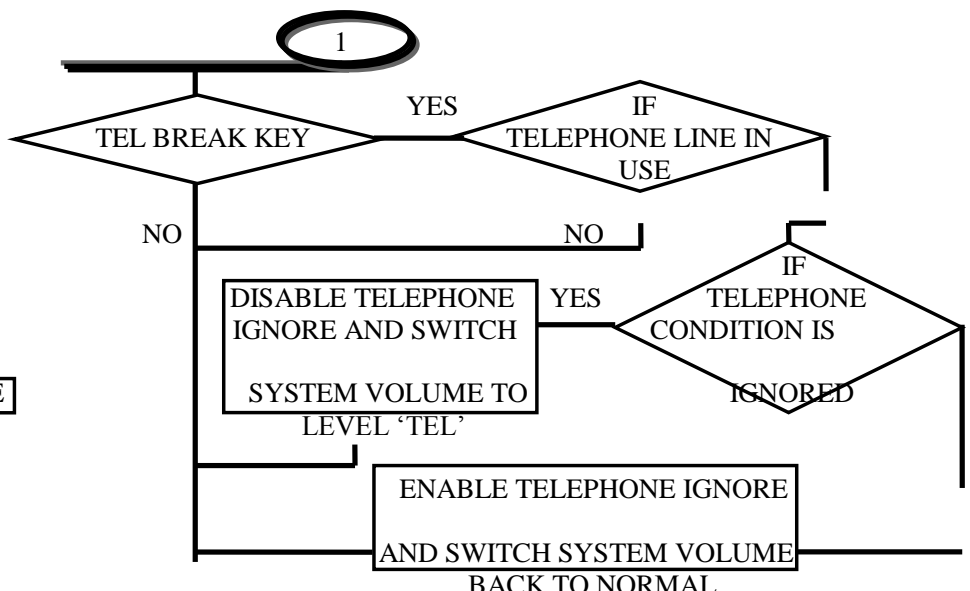

BACK TO NORMAL

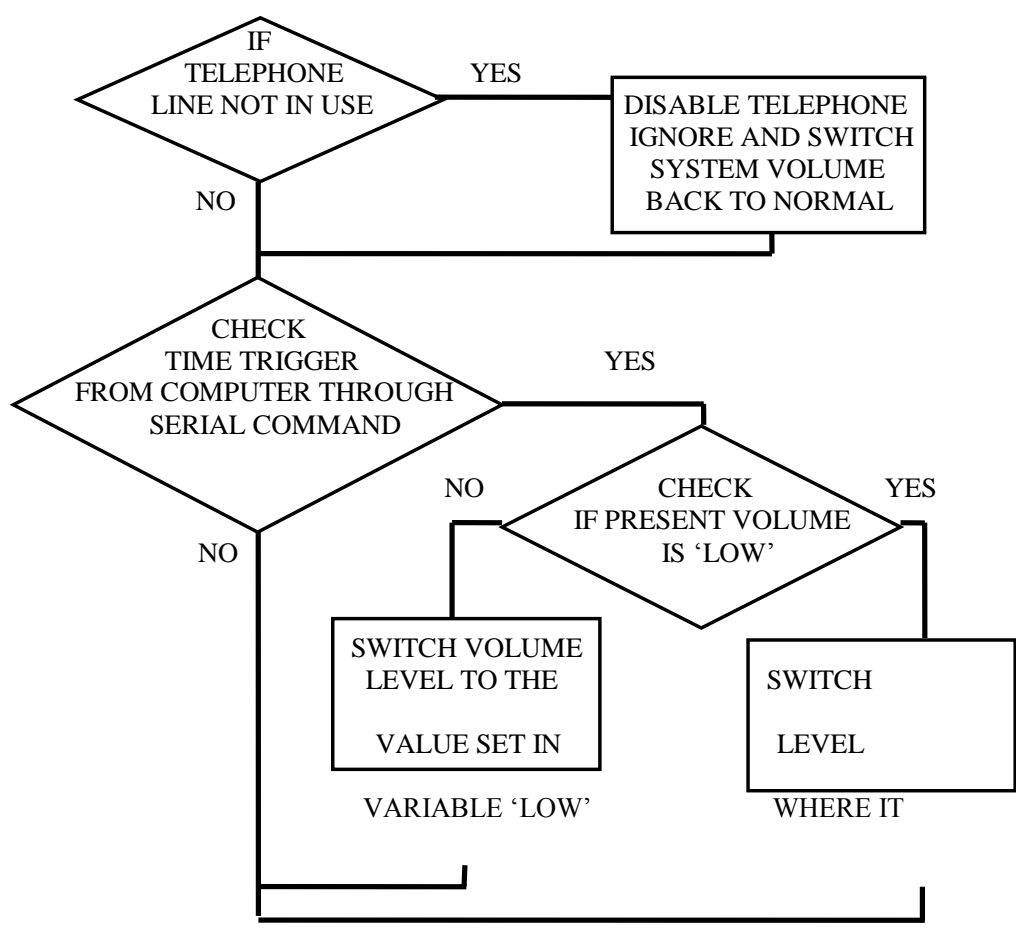

DISPLAY VOLUME LEVEL IN THE 7-SEGMENT

IN NORMAL CONDITION.

TOGGLE 'Dp' LED AND VOLUME LEVEL IN LOW VOLUME CONDITION.

TOGGLE ' $\mathrm{g}$ ' LED AND VOLUME LEVEL IN TELEPHONE ACTIVE CONDITION

SEND PRESENT VOLUME LEVEL OF THE SYSTEM, TO COMPUTER THROUGH SERIAL

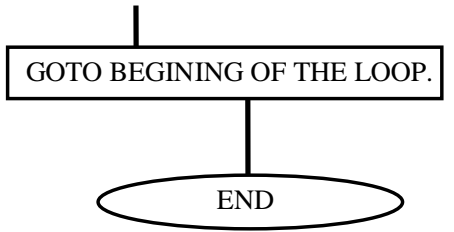




\section{FUTURE SCOPE AND CONCLUSION}

Following enhancements can be made individually on the hardware part and the software part as follows.

Hardware - In this device, the timer thing is implemented in the software part and the volume change is being performed in the hardware. The implementation of a hardware clock attached to this device can make this thing work independently. In that clock, alarm time can be set by the user and the sound level will go down accordingly. The clock can act as an alarm clock too on implementing a separate buzzer with it. Further implementation of an IR remote with this device can make it more efficient and help the user to control the device from a distance. The volume level a user sets in the system gets reset, once the device is restarted. Implementing, a memory, i.e. an EEPROM with the device can solve the problem.

Software - The software that helps the user to interact with the hardware can be made provided with more features, such as, a countdown timer which will also help in the alarm operation. A reminder facility to notify the user about his scheduled works can be implemented. A stopwatch can also be implemented along with the software. Provisions of setting an alarm with date and not only time can be implemented further.

This system came up as a need to a guy who is a music freak and is clumsy with his stuffs. In this project, microcontroller based intelligent digital volume controller with timer, we designed a hardware that is capable of controlling volume of any audio system and is also capable of switching volume levels during incoming or outgoing phone calls in wired network. The microcontroller programming is performed in Embedded C. Each and every operation in this device is made interactive and easy to recognize. The device surrenders itself to its user's will in case of volume levels. The computer software is designed in such a way, that it can be easily operated.

\section{ACKNOWLEDGMENTS}

The authors express a deep sense of gratitude to the Department of Computer Science, JIS College of Engineering, Kalyani, Nadia-741235, West Bengal, India and Department of Computer Science, Barrackpore Rastraguru Surendranath College, Kolkata-700 120, West Bengal, India for providing necessary support for the work and their family members for being constant inspiration and motivation for pursuing such works.

\section{REFERENCES}

[1] AT89S52 Datasheet, http://www.atmel.com/dyn/ resources/prod_documents/doc1919.pdf

[2] CD4066 Datasheet, http://members.shaw.ca/roma/ 4066.pdf

[3] Opto-Isolator, http://en.wikipedia.org/wiki/Opto-isolator

[4] Zener-Diode, http://en.wikipedia.org/wiki/Zener_diode

[5] D. Chattopadhyay, P.C. Rakshit, New Age International Publication, Electronics - Fundamentals and Applications p. 72

[6] Voltage divider or Potential divider, http://en.wikipedia.org/wiki/Voltage_divider

[7] Alarm \& Clock software from CNET, http://download.cnet.com/Alarm/3000-2350_410742679.html?tag=main;dropDownForm

[8] Cool Timer from Harmony Hollow software, http://www.harmonyhollow.net/cool_timer.shtml

[9] Time Left is a timer Software, http://www.timeleft.info/timer-software.html

[10] AVR CDC, http://www.recursion.jp/avrcde/

[11] LM317 Datasheet, http://www.ti.com/lit/ds/symlink/ $1 \mathrm{~m} 117 . \mathrm{pdf}$

[12] LM 317 Calculator, http://www.electronicslab.com/articles/LM317/

\section{ABOUT THE AUTHORS}

Diptarup Paul is presently working as a Hardware and Embedded System Research and Development Engineer in Security Engineers Private Limited, West Bengal, India. He has received his B. Tech in Computer Science from West Bengal University of Technology, West Bengal, India. His research interest includes Cryptography, Steganography, Microcontroller and Microprocessor, Biometric Sensors, RFID.

Sukalyan Som is presently working as an Assistant Professor in Computer Science in Barrackpore Rastraguru Surendranath College, West Bengal, India. He is having over 5 years of teaching experience. He has received his B.Sc. in Statistics from University of Calcutta, West Bengal India and Masters in Computer Application from West Bengal University of Technology, West Bengal, India. His research interest includes Cryptography, Steganography, Image Processing, Computational Geometry etc. 


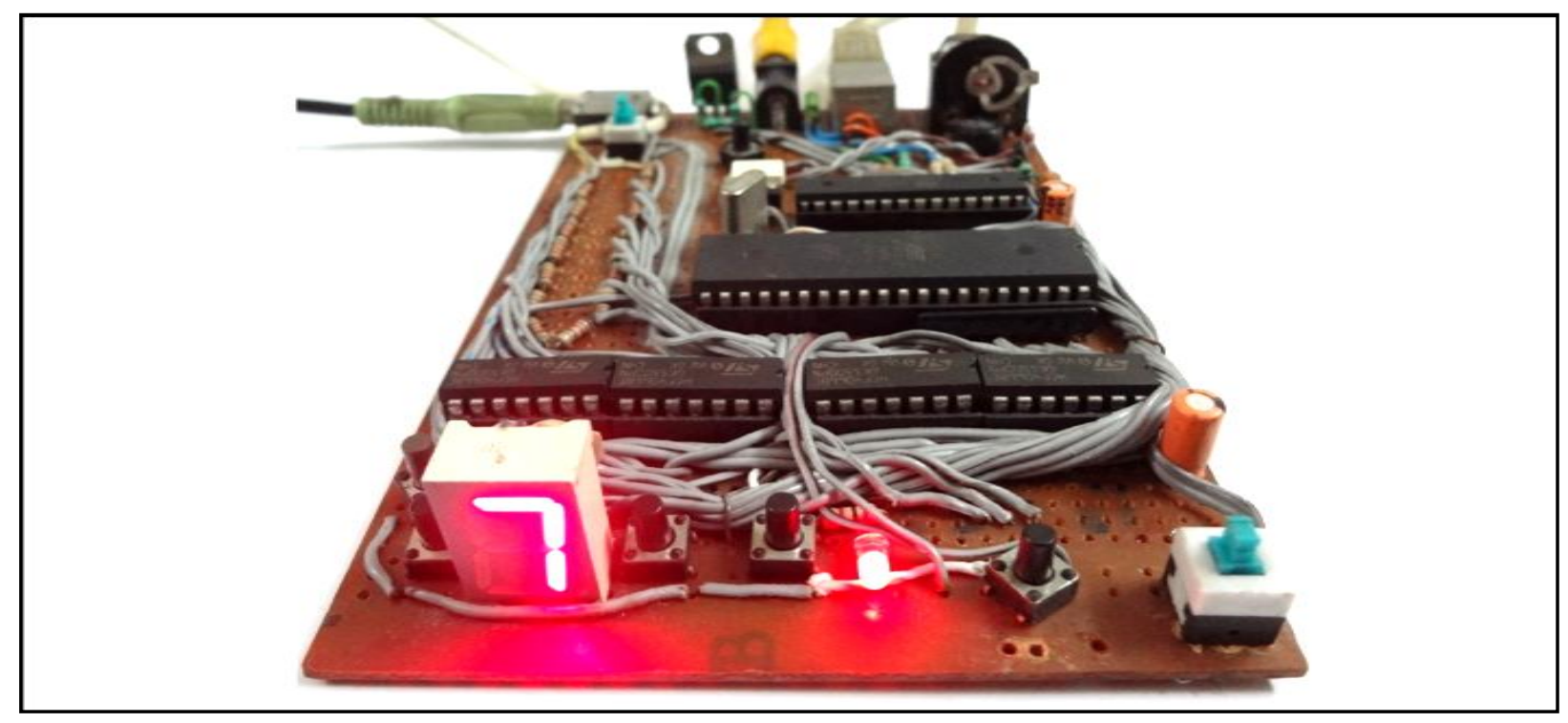

Fig 13: Microcontroller Based Intelligent Digital Volume Controller with Timer (Front View).

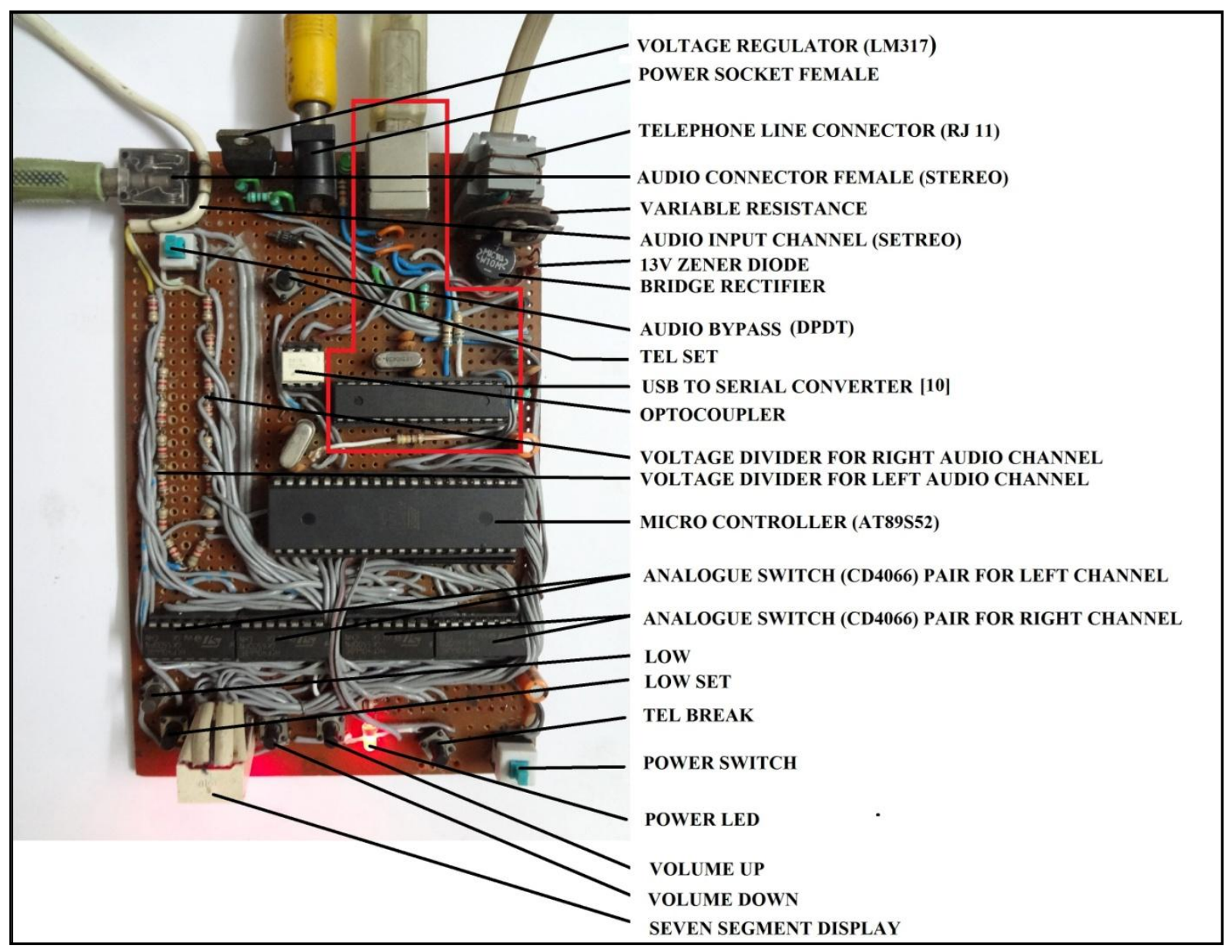

Fig 14: Microcontroller Based Intelligent Digital Volume Controller with Timer (Top View). 